\title{
Development of a Smart Meter, Power Line Communications Supported under loT Architecture
}

\author{
F. Juan Carlos Vesga*, H. Martha Fabiola Contreras and Gerardo Granados Acuña \\ Escuela de Ciencias Basicas Tecnologia e Ingenieria (ECBTI), Universidad Nacional Abierta y a Distancia; \\ Carrera 27 Nro.40-43. Bucaramanga,Colombia; juan.vesga@unad.edu.co, martha.contreras@unad.edu.co, \\ gerardo.granados@unad.edu.co
}

\begin{abstract}
Background/Objectives: Optimization of energy consumption has become the subject of ongoing research, in order to establish mechanisms that provide gradually to reduce global warming. In this paper the design, construction and implementation of a system of control and monitoring of power consumption (Smart Meter), supported on power line communications (PLC / Power Line Communications) under IoT architecture is proposed; which allows real-time measurements of various existing parameters in the power system at very low cost, using existing technology in the market. Methodology: To develop the prototype will use the grid as a physical transmission medium via a communication system HomePlug AV, a web server embedded under IP protocol for administering the communications system and control system power control comprised of a phase detector and zero crossing, an instrumentation system and a control system power supported state devices solid. Results: The developed prototype not only optimizes energy consumption of each electrical device, but also to measure in real time variables such as active power, reactive power and power factor; all operating on Internet Protocol (IP) and under using existing technology in the market. Relevance of the topic: The optimization of energy consumption is an issue of vital importance in the world and the development of this prototype raises the possibility of being implemented through the use of embedded systems of low cost and ease of implementation in homes in the future near. Application/Improvements: The developed prototype can be considered as a technological innovation strategy in the field of IoT, because it will allow to know in a very fast and efficient way the consumption of electrical energy product of a load, in terms of active power, reactive power, Parente power, power factor, RMS voltage and RMS current, variables of great importance in the residential or industrial context, which are quantified in an effective way depending on the waveforms and not through indirect methods such as performs conventionally on similar meters. In future related work it is recommended to establish mechanisms that allow to reduce the size of the hardware element, but also to make use of other alternative telecommunications technologies, in order to allow the development of low cost prototypes and ease of implementation.
\end{abstract}

Keywords: IoT, Optimization, Power, Power Consumption, PLC

\section{Introduction}

Currently, various organizations and users seek viable alternatives to optimize energy consumption in order to reduce costs and be consistent with governing environmental policies worldwide. Among the main alternatives can be mentioned: investment in technological equipment, improved management of energy resources,

${ }^{*}$ Author for correspondence 
establishing new work habits, consumption and organization. In most cases, the implementation of some of these strategies can be inefficient and costly during implementation.

Home automation is a field of engineering-oriented management of three major application areas are: comfort, security and energy management ${ }^{1}$. This subject is under study within technology Internet of Things (IoT), as well as the term "Smart Grid" which follows a concept oriented control, management and optimization of the grid 2 . The proposed system seeks to make use of a network infrastructure and protocols for specialized communication that allow the interconnection of a series of equipment and sensors, with the aim of obtaining realtime information on variables to control in the home environment, thereby generating actions on said chord setting with the results obtained in each of the measurements.

The objective of the system supported IoT is to use advanced information and communication technologies (ICTs) to control modern power systems reliably and efficiently ${ }^{\underline{3}}$. To do this, the tendency is for greater control of low voltage networks, by using "smart metering" (Smart
Meter) residential (smart homes) and enterprise environments (smart businesses) ${ }^{4}$ which significantly affects the network performance when the number of users increases.In light of the foregoing, it is proposed the use of the Smart Model (SM. One of the most interesting topics in the field of Smart Grid focuses on using the power grid as a physical transmission medium (Power Line Communications - PLC), from which it seeks to integrate systems and power control communications into one. Under this new application, the prototype should be able to maintain constant communication between systems management and user terminal devices, thereby enabling the creation of intelligent energy-saving systems in homes ${ }^{5}$.

\section{Methodology}

The use of energy and its proper and responsible management are the first steps to achieve considerable savings ${ }^{6}$. In order to obtain an effective plan to achieve energy savings it is necessary to know the entire workspace or housing. In view of the above, the next technological development based on the HomePlug standard, aimed at the optimiza-

Table 1. HomePlug AV HomePlug 1.0 vs

\begin{tabular}{|c|c|c|}
\hline Characteristic & HomePlug 1.0 & HomePlug AV \\
\hline Transmission speed & $14 \mathrm{Mbit} / \mathrm{s}$ & $200 \mathrm{Mbit} / \mathrm{s}$ \\
\hline Throughput (max) & $8 \mathrm{Mbps}$ & $120 \mathrm{Mbps}$ \\
\hline Throughput (Typical) & $4-6 \mathrm{Mbps}$ & $50-70 \mathrm{Mbps}$ \\
\hline Bandwidth & $4-21 \mathrm{MHz}$ & $1.8-30 \mathrm{MHz}$ \\
\hline Channel access (QoS) & CSMA, CA (4) & TDMA, CSMA (8) \\
\hline Modulation & DQPSK (OFDM) & $\begin{array}{c}\text { BPSK, QPSK, 16 QAM, 64 QAM, } \\
\text { 256 QAM, 1024 QAM (OFDM) }\end{array}$ \\
\hline Applications & Data & $\begin{array}{c}\text { Data, VoIP, Telephony, HDTV, } \\
\text { Home Theater }\end{array}$ \\
\hline
\end{tabular}


tion of power consumption, supported on IP and under Smart Grid architecture is proposed; which from now on will be called "Smart Meter" or its acronym SM (Smart Meter).

HomePlug Power line Alliance is an association of related industries PLC. In June 2001, the HomePlug standard v1.0 was launched ${ }^{7}$ Which allows speeds up to $14 \mathrm{Mbps}$. Currently, the most recent standard is the HomePlug AV2, which allows speeds up to $500 \mathrm{Mbps}$. It has a mechanism media access (MAC / Medium Access Control) function of a distributed coordination (DCF / distributed coordination Function) protocol based multiple random access carrier sense with collision avoidance (CSMA-CA / carrier sense multiple access with collision avoidance). HomePlug is considered one of the most important standards in PLC technology ${ }^{\underline{8}}$ which utilizes CSMA/CA as a mechanism to accessing the media. The main purpose of this paper is to assess the performance of a network supported in HPAV standard under the scheme AFR. Methods/Statistical Analysis: In this paper, the concept of saturation of throughput will be used for a finite number of stations. For that matter, it is necessary to assume that the size of the package is fixed of bits to a rate of Mbps. Additionally, an adaptation to Bianchi's model was carried out adjusted to the MAC structure of HPAV, which allows to represent CSMA/CA as a Markov's chain in discrete time of two dimensions. All this is done in order to represent the back-off procedure under a scheme DCF with saturated stations. Relevance of the Subject: AFR is a scheme that was proposed in order to achieve a high efficiency in the control layer of media access (MAC. In Table 1, a comparative table between the HomePlug 1.0 and HomePlug AV (HPAV) protocols is provided.

An additional element of the HPAV technology is its coexistence with HomePlug 1.0 devices and computers that provide access broadband over power lines of medium and low voltage. Furthermore, due to its widespread use, the HPAV devices and operating in hybrid configurations that facilitate the interconnection network and wireless PLC?.

With the development of Smart Grid systems for energy management and evolution of HPAV standard; in mid-2012 it was presented version Homeplug Green $\mathrm{PHY}^{\mathrm{Tw}}$ emerged as the leading specification to implement Smart Grid functionality in home area networks (HAN / Home Area Networks) based on PLC ${ }^{10}$. Table 2 shows

Table 2. HomePlug AV HomePlug Green PHY ${ }^{\text {to }}$ vs

\begin{tabular}{|c|c|c|}
\hline Characteristic & HPAV & HomePlug Green PHY $^{\mathrm{m}}$ \\
\hline Spectrum & $1.8 \mathrm{MHz}-30 \mathrm{MHz}$ & $1.8 \mathrm{MHz}-30 \mathrm{MHz}$ \\
\hline Modulation & OFDM & OFDM \\
\hline Number of subcarriers & 1155 & 1155 \\
\hline Space subcarrier & $24,414 \mathrm{kHz}$ & $24,414 \mathrm{kHz}$ \\
\hline Channel access & CSMA / CA, TDMA & CSMA / CA \\
\hline $\begin{array}{c}\text { Subcarrier modulation formats } \\
\text { supported }\end{array}$ & $\begin{array}{c}\text { BPSK, QPSK, } 16 \text { QAM, 64 QAM, 256 } \\
\text { QAM, } 1024 \text { QAM } \\
\text { only QPSK }\end{array}$ \\
\hline $\begin{array}{c}\text { Robust OFDM } \rightarrow 4 \text { to } 10 \mathrm{Mbps} \\
\text { Adaptive bit loading } \rightarrow 20 \mathrm{Mbps}-200 \\
\text { Mbps }\end{array}$ & $\begin{array}{r}\text { Robust OFDM } \\
4,5 \text { and } 10 \mathrm{Mbps}\end{array}$ \\
\hline
\end{tabular}


the technical features of the HPAV Vs HomePlug ${ }^{\text {mo }}$ Green PHY protocols are presented.

The main feature of HomePlug Green PHY ${ }^{\text {mw }}$ is that it is a version of low cost and low power consumption, adapted from HPAV, which allows interoperability with IEEE 1901 and HomePlug AV respectively, because only uses Shift Keying of cuadrafásica phase (QPSK / Quadrature phaseShift Keying) as a modulation technique within each carrier and multiplexing orthogonal frequency division (OFDM / orthogonal frequency division multiplexing) robust (ROBO) as a strategy for implementing a communications system stable, regardless of how hostile channel conditions are (mains) $)^{11}$.

\subsection{Proposed Architecture}

The prototype set designed for residential and small business environments, operates from a communications network based on PLC supported on the HomePlug AV standard, accompanied by an interface for power devices (appliances) and data devices (PCs, printers, etc.). In Figure 1 the proposal in residential context architecture is presented.
The prototype developed or Smart Meter (SM), is the main element of the architecture, which is composed of three areas: a PLC adapter in order to make use of the electricity network as a physical transmission medium, a web server embedded low IP protocol responsible for managing the communications system and control, and finally, a system power control comprised of a phase detector and zero crossing, an instrumentation system and a control system power supported on solid state devices. Before connecting the SM to the corresponding power network configuration must be done to the device IP address. Later, you can send and receive data over the mains under the HomePlug standard.

The information generated by the SM, from the embedded system can be processed using a local server or a remote server. Among the many functions performed on the server are the following:

- Monitoring charge (voltage parameters, power, current)

- Command sending-off charge to SM.

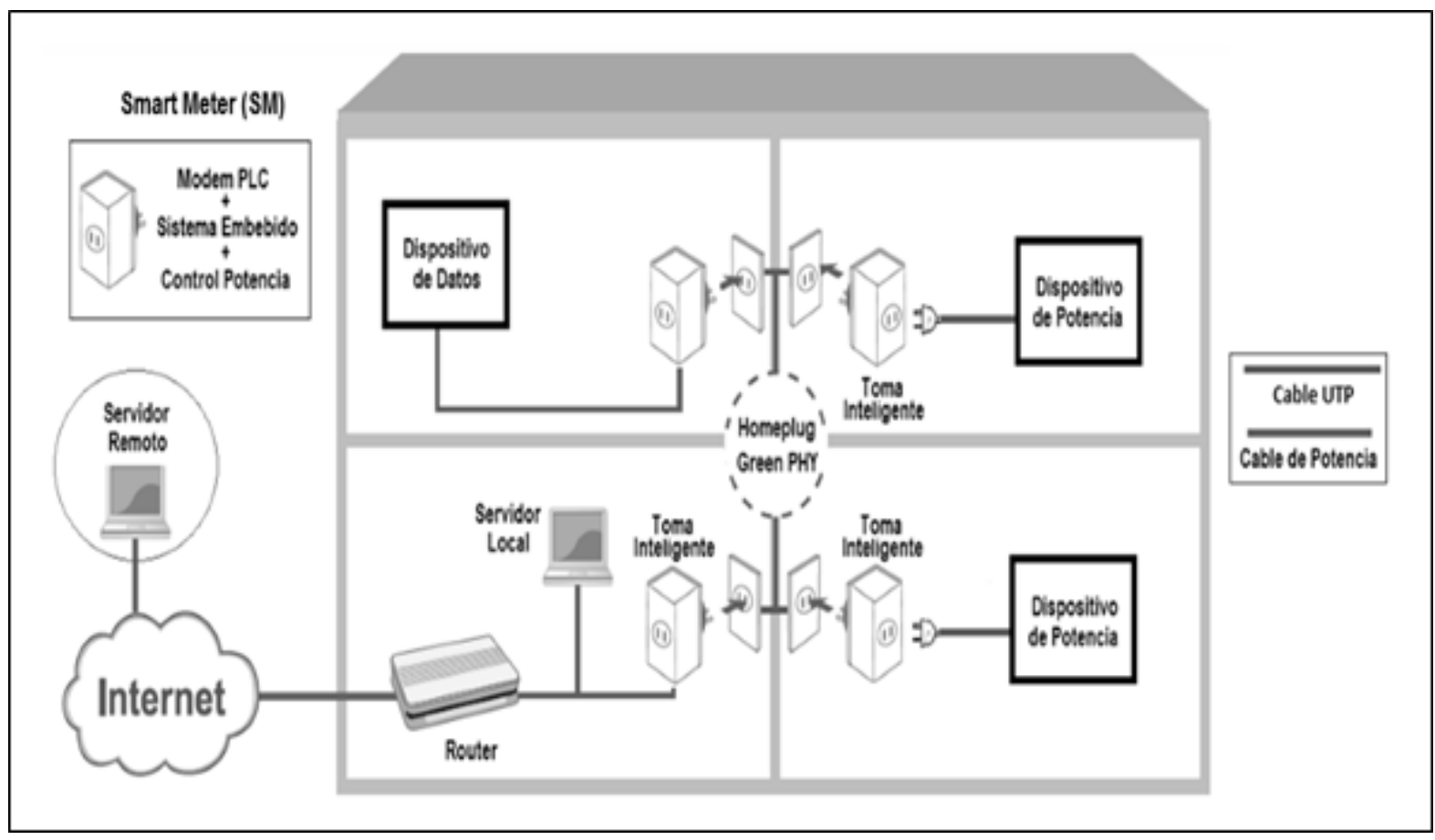

Figure 1. Outline of a System Power Control under IoT architecture. 
- Performance statistics generation and load consumption

- IP address management of SM forming part of the control system.

Using a remote server implies the need for a security system based on passwords and access permissions, in order to prevent malicious use of the system.

\subsection{Internal Architecture SM}

As mentioned above, the SM is basically comprised of three areas (A PLC communication system, an embedded Web server and a system power control). In Figure 2 corresponding to the internal architecture of the SM scheme is presented.

\subsubsection{Communication System with the Mains}

This system is the use of a PLC adapter model TP-LINK TL-PA211 AV200 (TP-LINK Industries, 2013). Table 3 including each of the technical specifications of the PLC adapter used.

\subsubsection{Central Processing System}

This system consists of an embedded Web Server reference SP1 - Server OEM (Net Media) SitePlayer HTTP Web, which can be programmed using hybrid form assembler programming languages, Java Script and HTML. Figure 3 presents the Modulo SP1 - SitePlayer HTTP Web Server OEM.

SitePlayer (SP), is an electronic circuit of reduced dimensions that houses inside full Web Server. It also has $48 \mathrm{~KB}$ of flash memory space to store custom Web pages, has 8 input output pins, a serial port and an Ethernet port. It supports ARP, ICMP, IP, UDP, TCP and DHCP protocols. The firmware is upgradable from the Ethernet connection, like updating the web pages that will be stored inside. It requires a $5 \mathrm{~V}$ supply voltage and consumes $75 \mathrm{~mA}$ current.

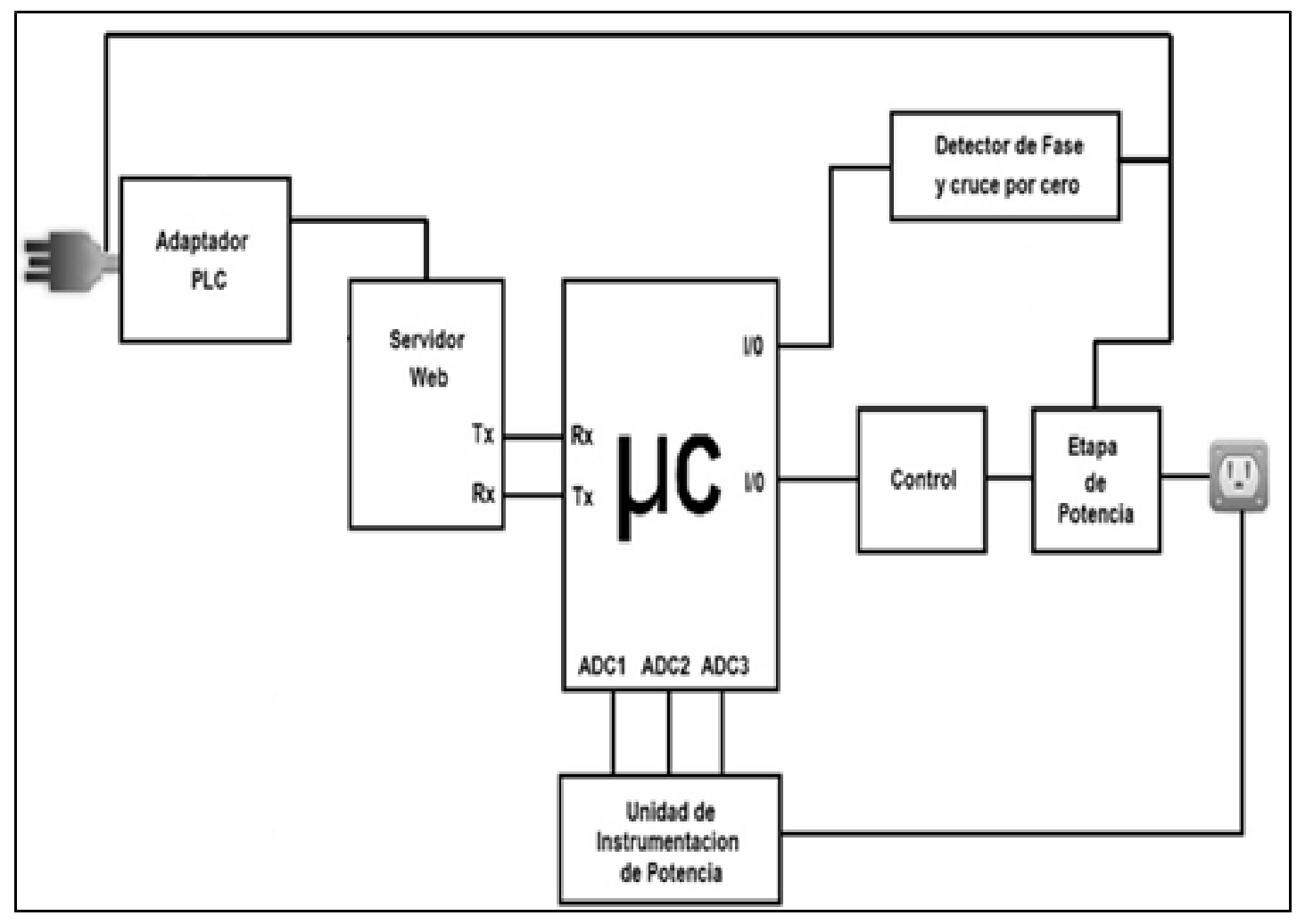

Figure 2. Block Diagram Smart Meter (SM). 
Table 3. Team TP-LINK TL-PA211 AV200 - Technical Specifications

\begin{tabular}{|c|c|}
\hline Characteristic & Value \\
\hline Standards and protocols supported & HomePlug AV, IEEE802.3, IEEE802,3u \\
\hline Interface & EU, US, UK, AU \\
\hline LED indicators & PWR, PLC, ETH \\
\hline Power consumption & $<3 \mathrm{~W}$ \\
\hline Modulation & OFDM \\
\hline advanced features & $\begin{array}{l}\text { Intelligent channel adaptation, } \\
\text { Integrated QoS Factory }\end{array}$ \\
\hline encryption & 128-bit AED \\
\hline Dimensions (W x D x H) & $93 \mathrm{~mm} \times 56 \mathrm{~mm} \times 30 \mathrm{~mm}$ \\
\hline Weight & 103 grams \\
\hline
\end{tabular}

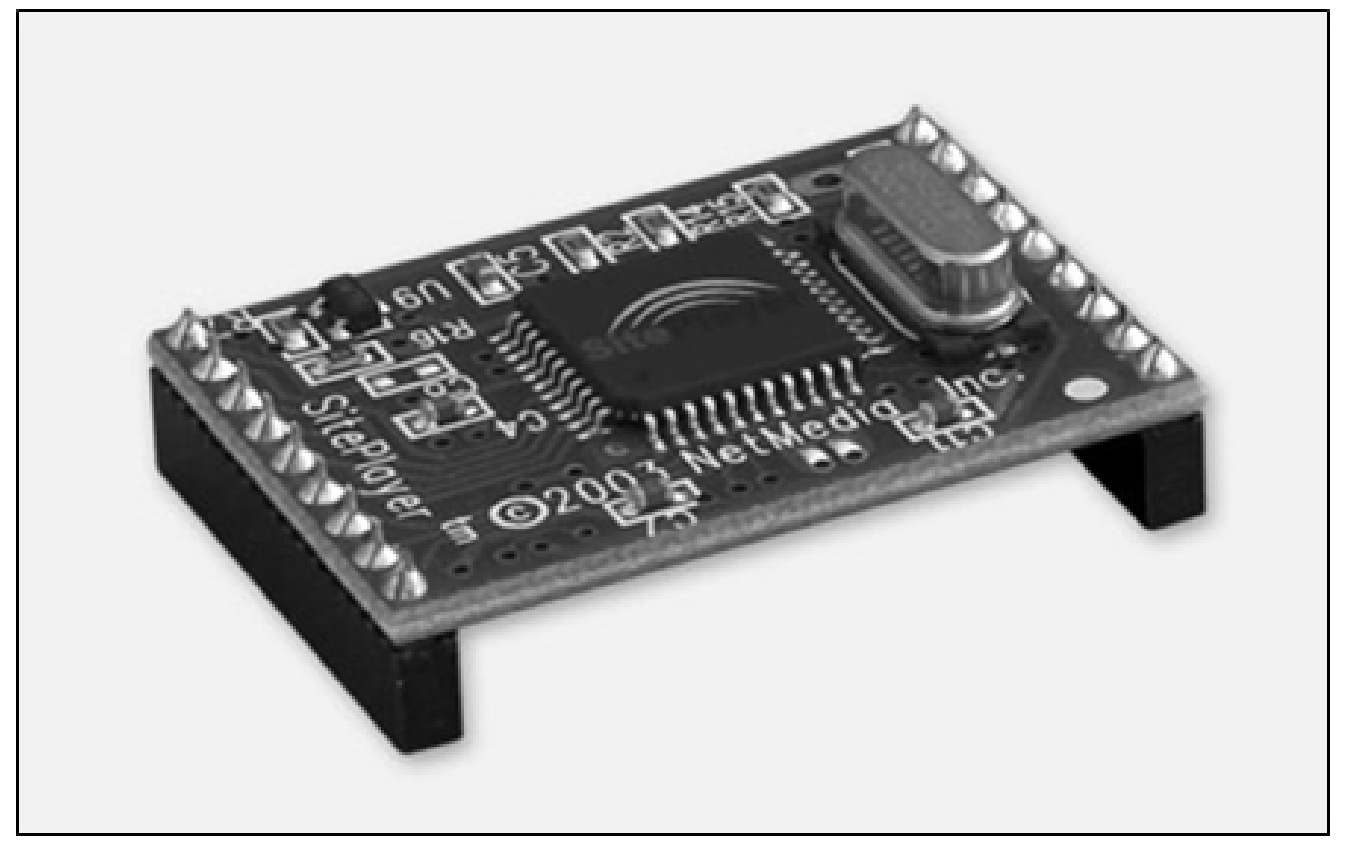

Figure 3. Module SP1 - SitePlayer HTTP Web Server OEM.

\section{Results}

With the information provided in the previous item, we proceeded to the development of Smart Meter includ- ing SitePlayer module and associated programming. In Figure 4 the module developed stands out.

Thanks to the services offered by the said device, a web application using HTML and Java Script, capable 


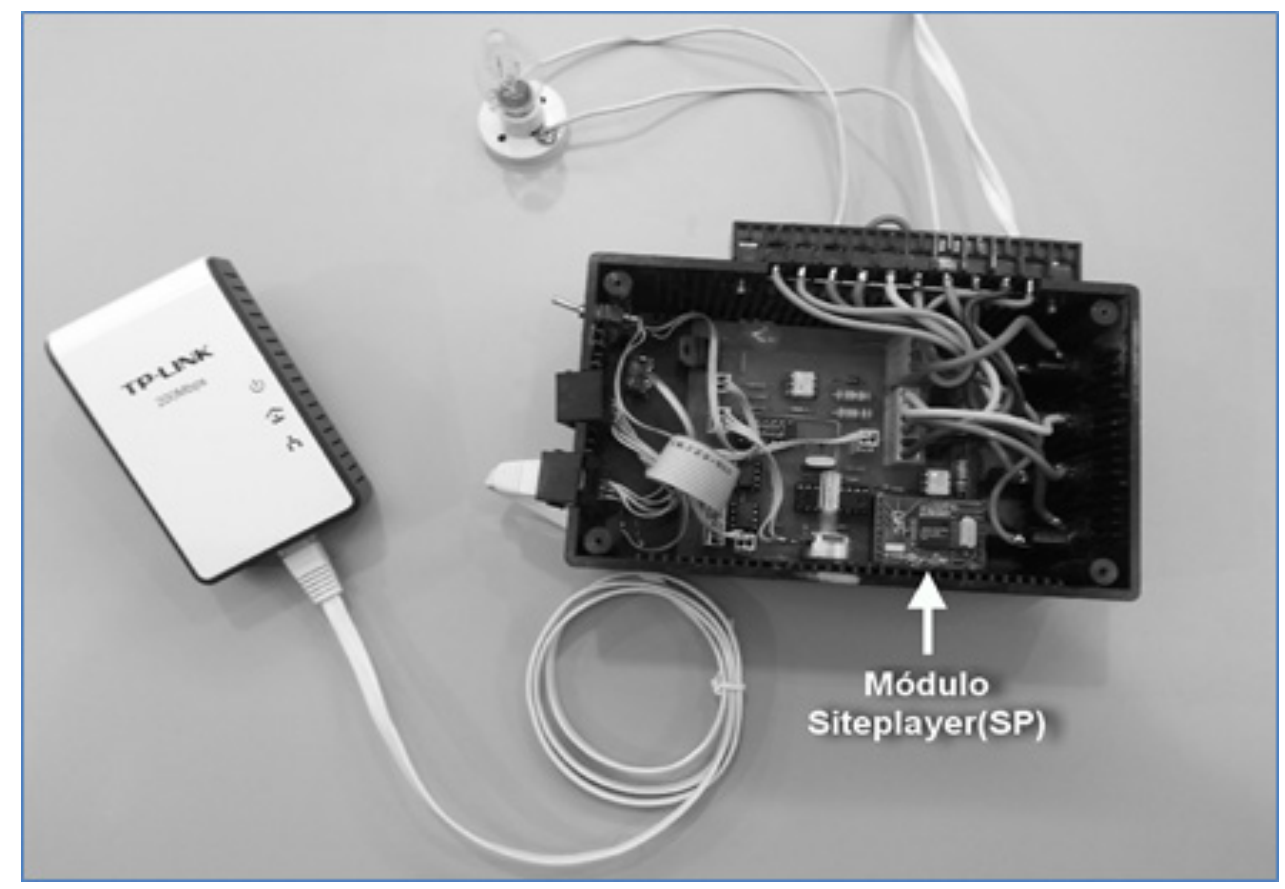

Figure 4. Construction of Smart Meter (SM).

of delivering real-time process control, measurement and ongoing monitoring of the energy consumed by a particular device under Smart Grid architecture devel- oped. Moreover, the inclusion of a microcontroller in the process control and instrumentation was necessary, because the SitePlayer not have A / D converter or exter-

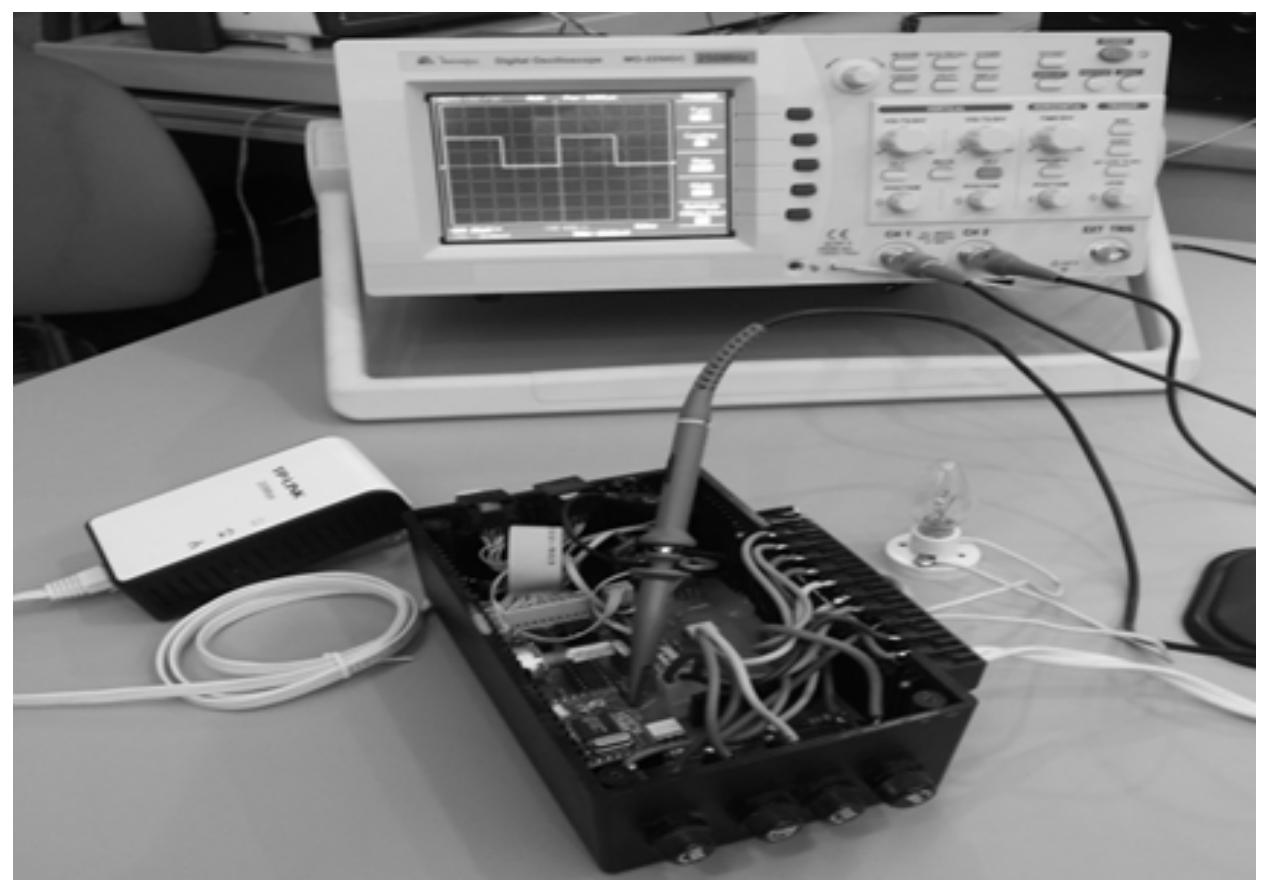

Figure 5. Smart Meter test (SM). 
nal interrupt sources. Under the above, the SP provides a process of continuous serial communication with the microcontroller at 9600 baud and a process of permanent communication with the other SMs and the main server as part of the LAN supported on TCP / IP, using grid as physical transmission medium under the HomePlug standard. Some of the tests carried out can be evidenced in Figure 5 , in which the waveform generated by the phase detector and zero crossing inside the SM module can be appreciated.

To access the web application SM simply uses a web browser and enter the IP address of the SM to which you want to access. This action automatically redirects the browser to the index.html page, which is stored in the SP. an example corresponding to the "Energy" where power consumption is displayed at present in the SM at a given instant option form is presented in Figure 6. There is observed that it is possible to monitor the following variables: load current, phase voltage, active power, reactive and apparent power factor; which it is reflected that the SM is a very important tool, which allows to know the energy consumption by the load losses that may be presenting, among other factors; in real time, Smart Grid architecture low and very low cost of implementation. Additionally, this form includes a Dimmer function, which allows the user to set the desired percentage of energy supply to the load, which can properly manage energy consumption.

$<$ insert figure 6 here >

Figure 7 presents a form which can be administered in energy control, connection status and activation Dimmer function (gradual power supply) on the load. The application software is implemented using programming languages three interacting simultaneously embedded into the Web Server way. (HTML, Java Script and Assembler), with a maximum size of program memory of $48 \mathrm{~KB}$, according to manufacturer's guidelines, which is a great challenge considering that many functions and components cannot be used because it must be conditioned to SitePlayer embedded web server.

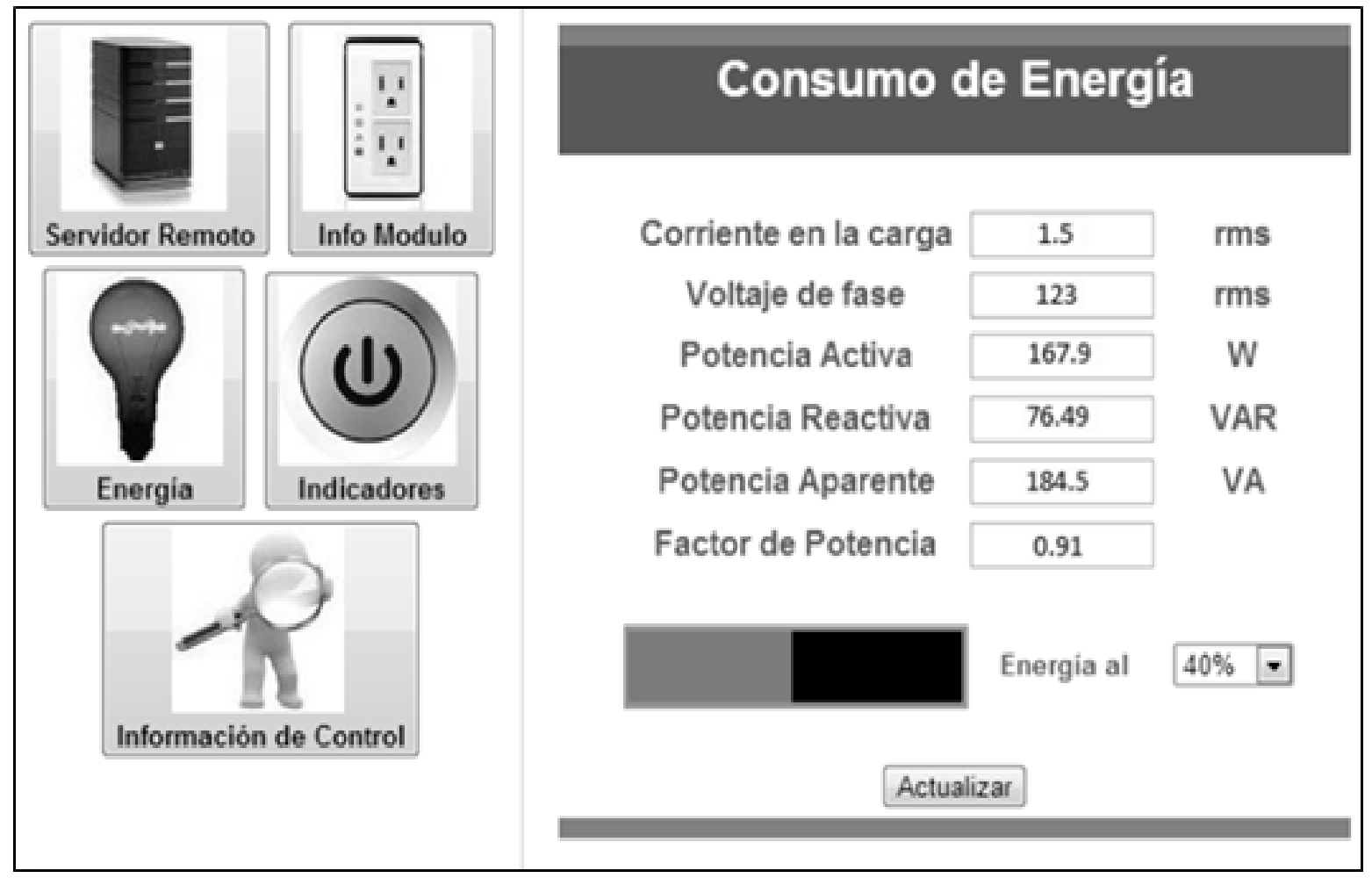

Figure 6. Monitoring and control form of energy. 


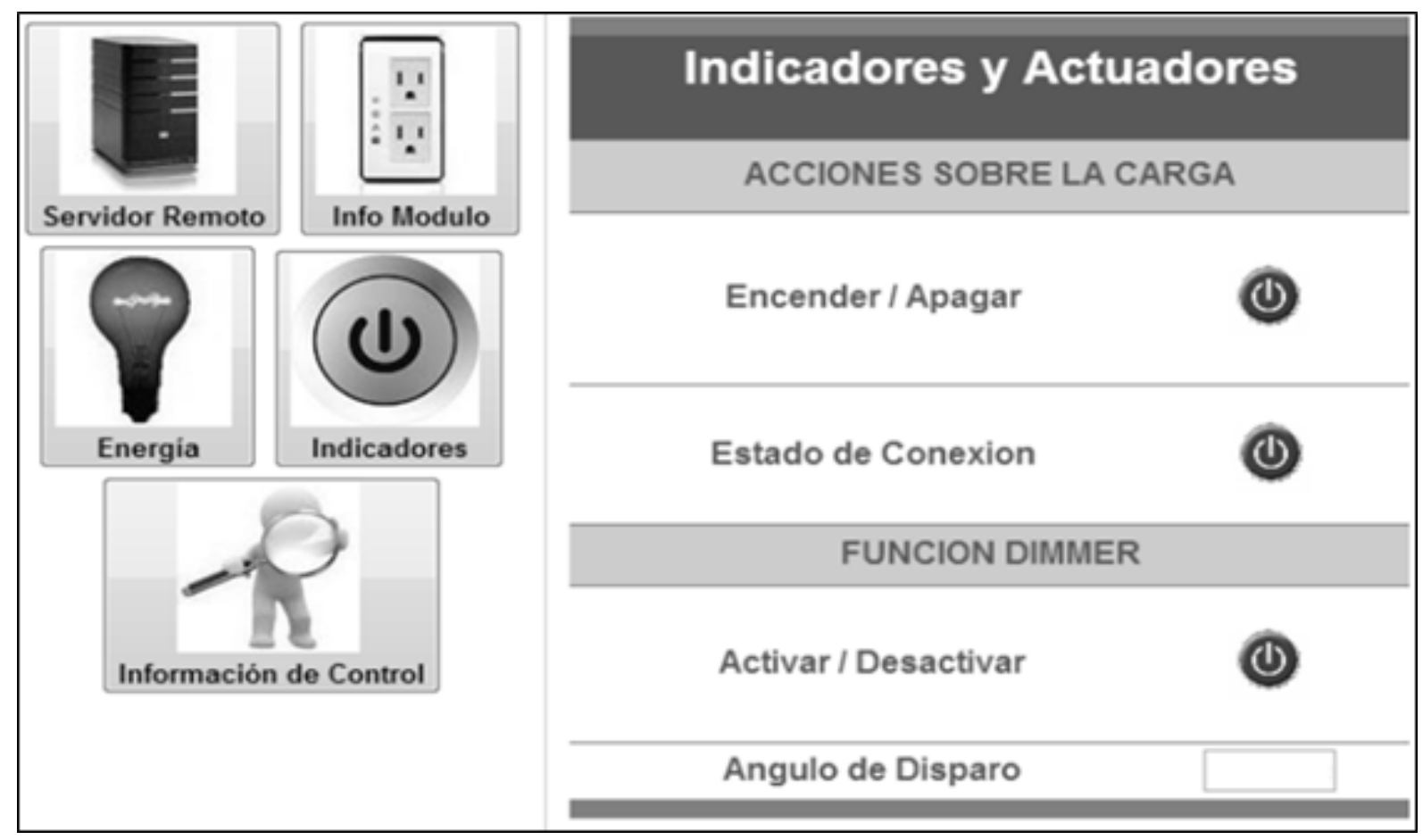

Figure 7. Power Control Form "Indicators and Actuators".

\subsection{Power Control on the Use of Solid State Devices}

In order to implement a system free from mechanical contacts, which with time tend to fail, a system for power control based on the use of solid state devices designed load. Then they describe each of the steps that are part of the system:

\subsubsection{Phase Detector and Zero Crossing}

The phase detector and zero crossing is a circuit that is responsible for identifying the state of the signal present on the mains, in order to establish the instants at which the power signal performs the process called "Crossing zero ", where the waveform changes from positive half cycle $60 \mathrm{~Hz}$ to negative and vice versa ${ }^{12}$ the Home Plug for Audio-Video (HPAV. The signal conditioning circuit allows $60 \mathrm{~Hz}$ into a square wave, which has the same frequency of the grid and edge detection microcontroller achieves synchronized with the mains.
The phase detector is comprised of a voltage divider as shown in Figure 8, through a 100k resistor (which carries most of the voltage) and a Zener diode $5.1 \mathrm{~V}$, which during the positive half cycle behaves as a $5 \mathrm{~V}$ regulator and during the negative half cycle behaves as a short circuit.

On leaving the opto transistor has a square signal $5 \mathrm{v}$ to $60 \mathrm{~Hz}$, which is sent directly to the microcontroller to be analyzed and accurately quantify the firing angle desired for the triacs. The wave is applied to pin RB0 microcontroller, which by means of the external interrupt detects rising or falling edges of the signal, thereby identifying the start of each half cycle. Present in the Zener voltage is applied to a series circuit composed of a resistance of $220 \Omega$ and an LED belonging to an opto transistor. When the Zener voltage is $5 \mathrm{~V}$, the LED lights opto transistor, causing the transistor between saturation mode and the output voltage is $5 \mathrm{~V}$. When the Zener voltage is $0 \mathrm{~V}$, the LED turns off opto transistor, 


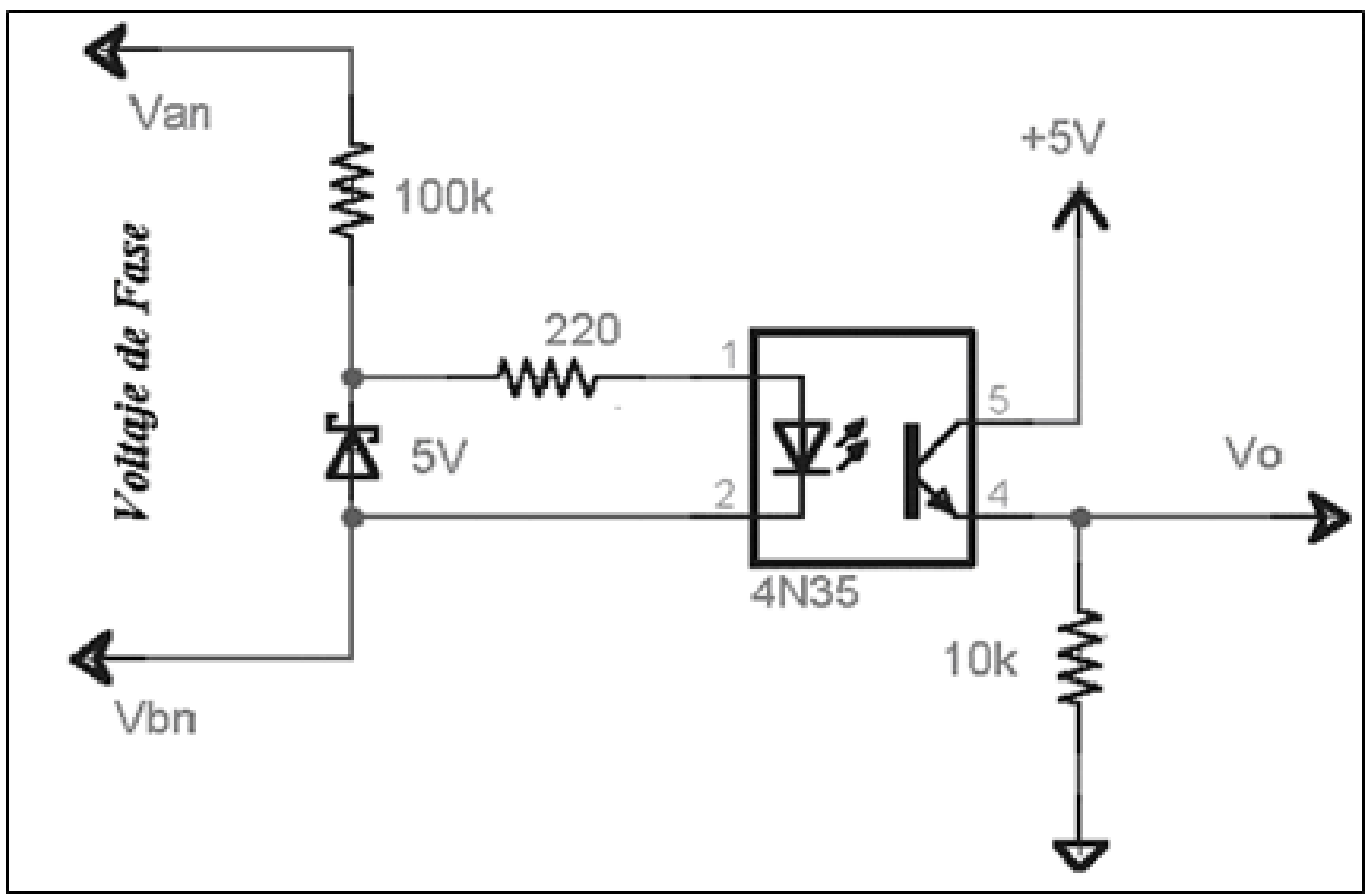

Figure 8. Phase detector and zero crossing.

This circuit provides a signal to $5 \mathrm{Vpp}$ opto transistor, which isolates the signal from the power supply of the control circuits. In Figure 9, the signals on the phase detector shown and zero crossing. Note that in this circuit two interesting things happen: The time corresponding to the high and low levels in the output

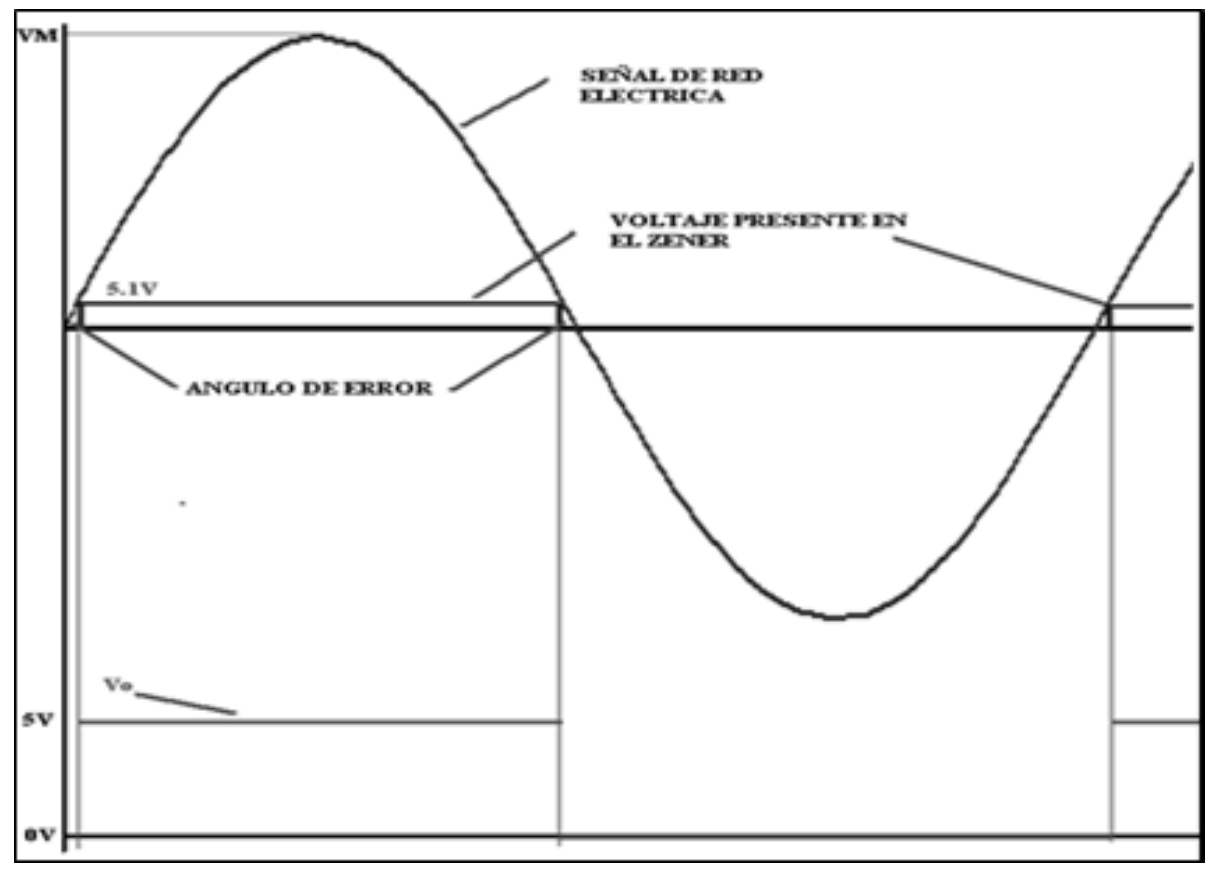

Figure 9. Waveforms present at the phase detector and zero crossing. 
voltage of the phase detector is not equal, leading to a small error in the firing of the triacs. The mentioned error due to the time it takes to reach its Zener regulated voltage, which can be approximated instant at which the instantaneous phase voltage $5.1 \mathrm{~V}$ equals. This error is actually reflected in an angle error calculated by equations (1) and (2):

$$
\begin{aligned}
& 5,1=V_{M} \sin (\emptyset) \\
& \emptyset=\arcsin \left(\frac{5,1}{V_{M}}\right)=\emptyset_{\text {ERROR }}
\end{aligned}
$$

In general cases we can assume a $\mathrm{VM}=170 \mathrm{~V}$, and replacing in equation (2) we obtain the equation (3):

$$
\emptyset=\arcsin \left(\frac{5,1}{170}\right)=1,72^{\circ}=\emptyset_{\text {ERROR }}
$$

It is indicating that the error in the firing angle of a triac can be considered negligible. Since for the positive half cycle, the angle error will be $1,72^{\circ}$ and the negative half cycle will $-1,72^{\circ}$.

\subsubsection{Interface for Activation of a Triac}

This circuit, which can be seen in Figure 10, is used to activate the TRIAC at a given instant of time from the microcontroller, realizing the coupling between this and the power section using an opto triac. This system in a real scenario proved to be quite stable and immune to erroneous activations caused by transients existing in the grid.

The trigger pulse which is applied to this circuit comes from the microcontroller and lasts a degree $(16,66 \mathrm{~ms} /$ 360 ), equivalent to $50 \mu$ s approximately. In the microcontroller routines for activating the TRIAC to a specific shot angle is configured, which act synchronously with the phase detector and zero crossing. The instant in which the firing angle equivalent to the time expires, a pulse duration applied $50 \mu$ s through corresponding pin and activating the TRIAC thereby permitting current flow through the load. By this system configuration functions $\mathrm{ON} / \mathrm{OFF}$ and Dimmer mode it is possible.

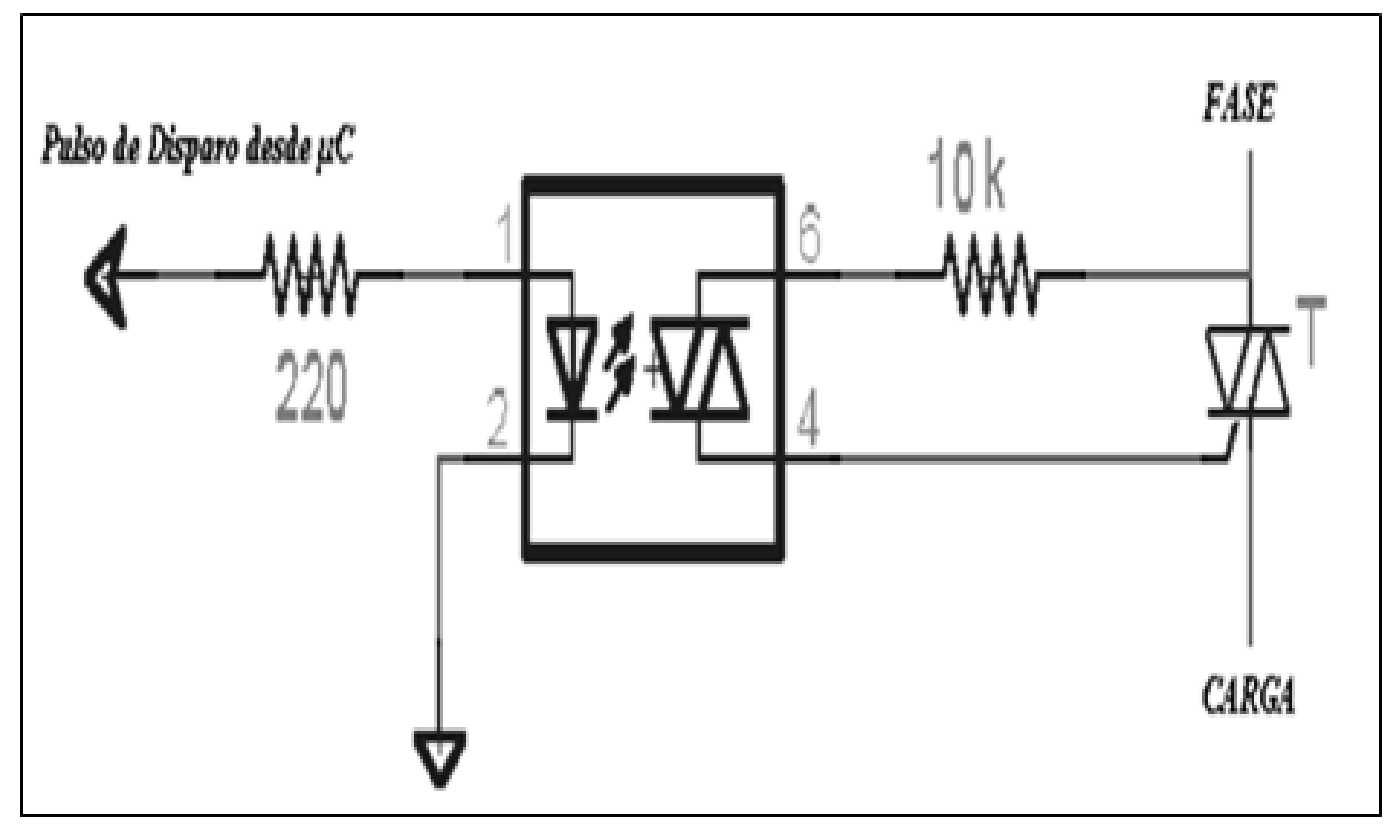

Figure 10. Triac firing circuit. 


\subsection{Power Measurement}

Electric power is defined as the ratio between the energy per unit time and is expressed in Watts. The energy consumed by an electrical device is called "Active Power", it expressed in kilowatt-hours (kWh) and corresponds to the value of power energy companies billed to each user. An associated power consumption element is called "Power Factor where the closer is its value to the unit, the greater the efficiency of the electrical system. The $\varphi$ angle corresponds to the angle existing phase between the voltage and current present in load $\underline{13}$.

The mathematical expression that describes the behavior of the electric power versus time is shown in equation (4):

$$
P(t)=V I \cos (\varphi)-V I \cos (2 w t-\varphi)
$$

Where :
$V$ : RMS potential difference between the terminals of the load

$I$ : RMS value of the current passing through the load

$V I \cos (\varphi):$ It is defined as Active Power

$V I \cos (2 w t-\varphi):$ It is defined as Fluctuating power, its average value is zero.

Another type of power present in the electrical systems is the reactive power, which is a type of power necessary to create magnetic fields by capacitive and inductive elements. This type of power is found by power companies as losses, this type of power does not produce useful work, is represented by the letter Q and measured in volt-amperes reactive (VAR) 14 .

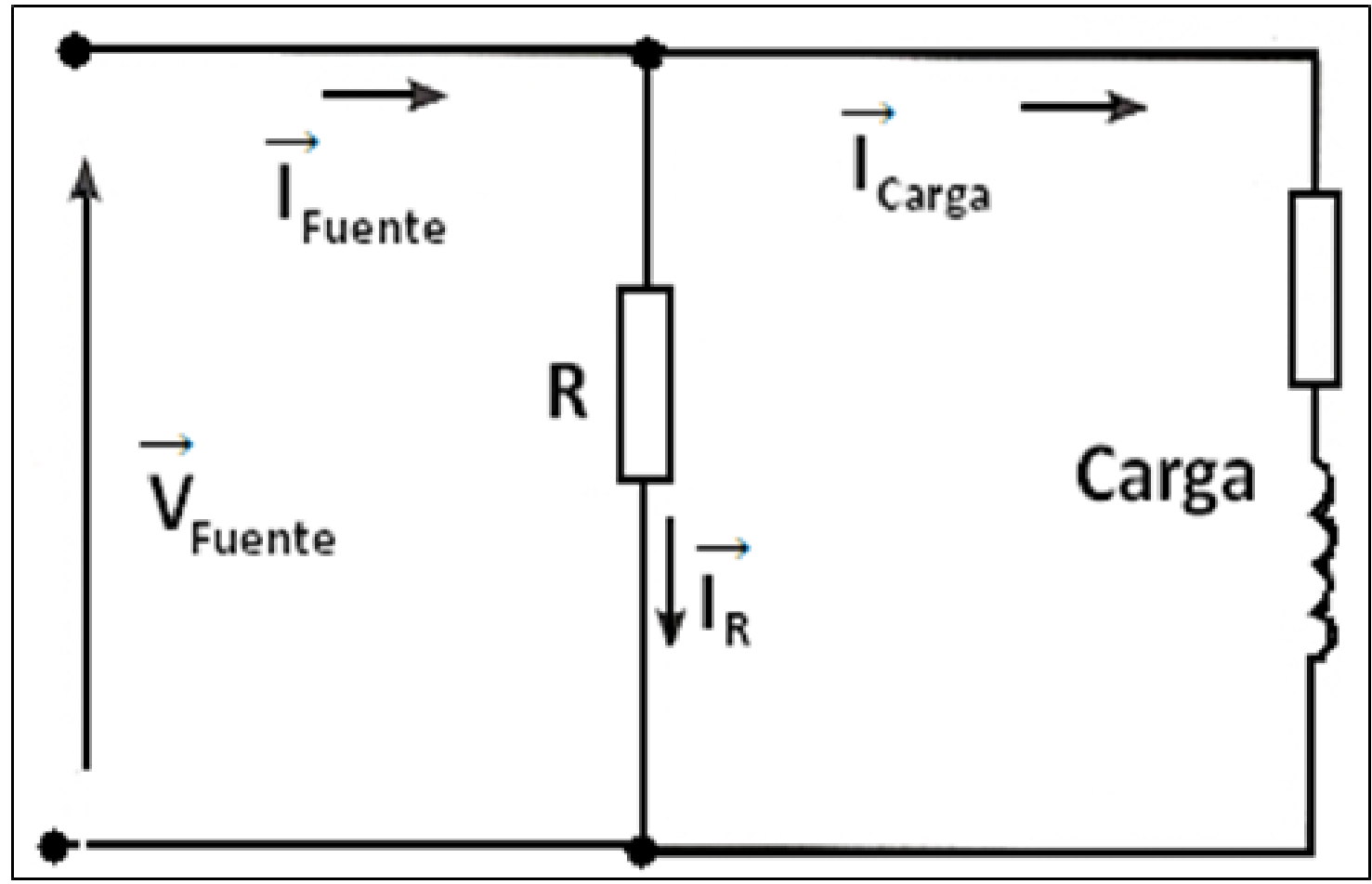

Figure 11. Connection scheme for measuring parameters in the load. 


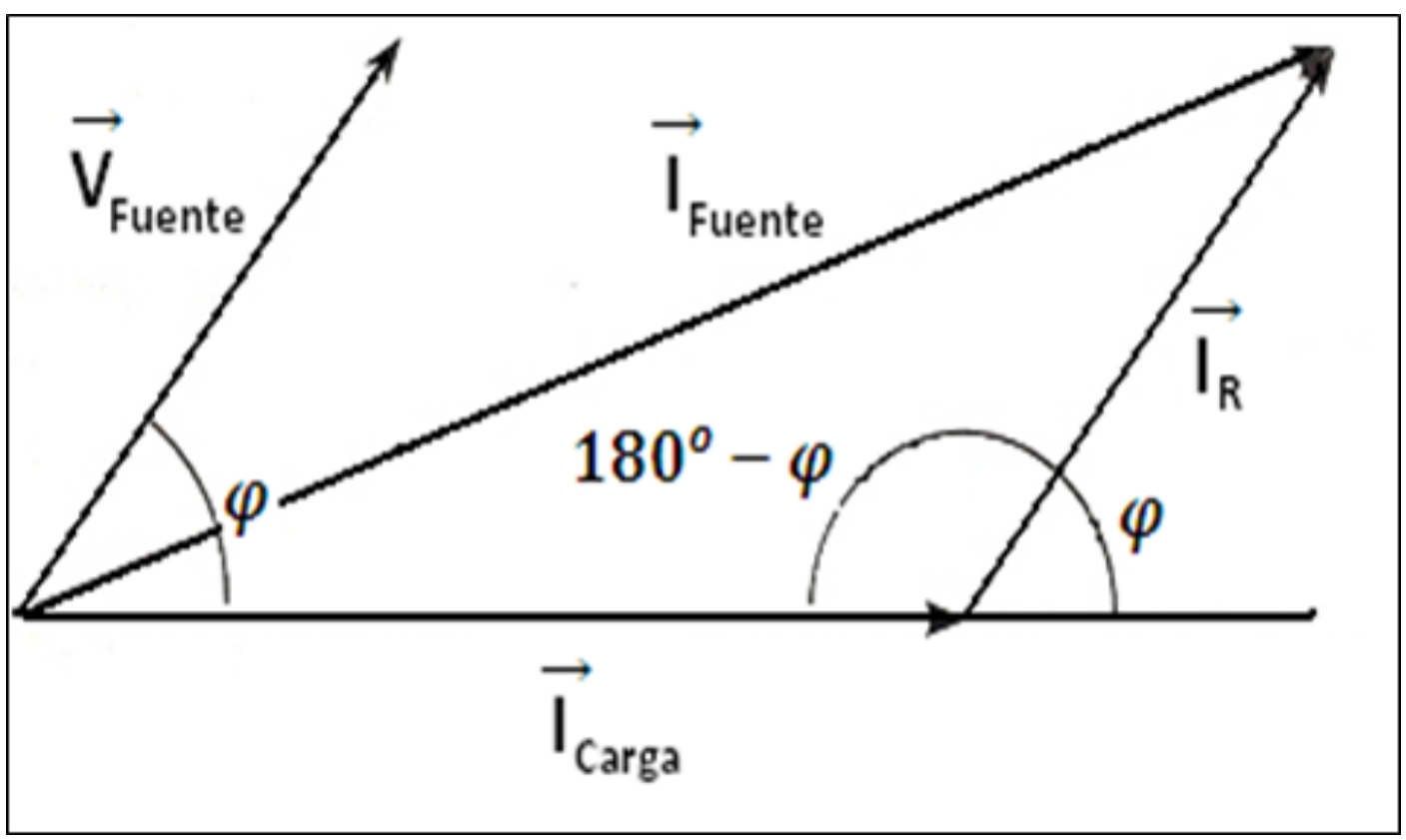

Figure 12. Phasor voltage and currents present in the charge.

One of the benefits the SM presents, it is possible to quantify the power factor directly on the load. In most cases, if you want to measure the power factor it is necessary to use robust measurement equipment and high cost, which makes impractical the use of this equipment at each desired point. In view of the above, a system that calculates not only the power factor on the load, but also allows indirect measurement of various parameters in the power system, which facilitates the process of power control is implemented through intelligent outlet and thus know firsthand the state of energy consumption and losses that may be presented in each outlet in real time, and very low cost of implementation.), Which must have a high ohmic value in order to minimize high current values that flow through it. As it is shown in Figure 11.

Given that the values of current and voltage can be represented in a phasor on systems AC, the values of currents and voltages present in the load resistance of reference and source can be represented by the phasor diagram as evidence in Figure 12.

According to Kirchhoff's current law have the equation (5):

$$
\vec{I}_{\text {fuente }}=\vec{I}_{\cdot_{R}}+\vec{I}_{\text {rearga }}
$$

Then, using the cosine theorem, equations (6-8) are obtained:

$$
I_{\text {Fuente }}^{2}=I_{R}^{2}+I_{\text {Carga }}^{2}-I_{R} I_{\text {Carga }} \cos (180-\varphi)
$$

$$
I_{\text {Fuente }}^{2}=I_{R}^{2}+I_{\text {Carga }}^{2}+I_{R} I_{\text {Carga }} \cos (\varphi)
$$

$$
\frac{I_{\text {Fuente }}^{2}-I_{R}^{2}-I_{\text {Carga }}^{2}}{I_{R} I_{\text {Carga }}}=\cos (\varphi)
$$

Where it corresponds to the power factor. Setting this value, it is possible to calculate the active, reactive and apparent in the load, which can be displayed simultaneously with values of voltage and current RMS present in the power system $\cos (\varphi)^{15}$. The expressions for calculating power values are: 
Active in load power (power consumption):

$$
\mathrm{P}_{\text {Carga }}=\mathrm{V}_{\text {Fuente }} \mathrm{I}_{\text {Carga }} \cos (\varphi)
$$

Reactive power load (losses):

$$
\mathrm{P}_{\text {Carga }}=\mathrm{V}_{\text {Fuente }} \mathrm{I}_{\text {Carga }} \operatorname{Sin}(\varphi)
$$

Apparent power :

$$
\mathrm{S}_{\text {Carga }}=\mathrm{V}_{\text {Fuente }} \mathrm{I}_{\text {Carga }}
$$

For the development of MS was used the sensor ACS714 Hall effect, which is designed to measure AC or DC current up to $5 \mathrm{~A}$, applications are varied, ranging from industry to academic projects. In this case three current sensors are required, which allow the calculation of power factor and other parameters mentioned above.

\section{Conclusions}

IoT has evolved from the convergence of wireless technologies, Micro-Electromechanical Systems (MEMS), micro-services and internet. Convergence has helped break down the walls of silos between Operational Technology (OT) and Information Technology (IT), allowing unstructured data generated by machines are analyzed for information that drives improvements. Based on the results it was verified that el Smart Meter developed a prototype low-cost, fully functional under an environment Smart Grid on IoT, which offers several advantages compared to other existing developments, considering that not only optimizes the energy consumption of each electrical device but also to measure in real time variables such as active power, reactive power and power factor; All about IP and under using existing technology in the market.

\section{References}

1. Sierra JE, Carlos-Vesga J, Medina B. Livestock model innovation under the concept of smart cities. Indian Journal of Science and Technology. 2018; 11(22):1-6. https://doi. org/10.17485/ijst/2018/v11i22/122510.

2. Sanduleac M. Unbundled Smart meters in the new smart grid era: Assessment on compatibility with European standardisation efforts and with IoT features. 19th IEEE Mediterranean Electrotechnical Conference (MELECON); 2018. p. 35-41. https://doi.org/10.1109/ MELCON.2018.8379064.

3. Garai A, Attila A, Pentek I. Cognitive Telemedicine IoT technology for dynamically adaptive reference eHealth content management framework embedded in cloud architecture. 7th IEEE International Conference on Cognitive Infocommunications (CogInfoCom); 2016. p. 000187-000192. https://doi.org/10.1109/ CogInfoCom.2016.7804547.

4. Vesga JC, Sierra JE, Granados G. Evaluation of Hybrid models $(\mathrm{MH})$ and Smart (MS) based on game theory for resources allocation in HomePlugAV networks. Wseas Transactions On Communications. 2016; 15:1-17.

5. Sendin A, Gomez JS, Urrutia I. Large-scale PLC Gateway-based architecture for smart metering deployments. IEEE International Symposium on Power Line Communications and Its Applications (ISPLC); 2018. p. $1-6$.

6. Asset Management Conference (AM 2016). IET Conference Publications 710 [Internet]. [cited2016 Nov 23]. Available from: http://toc.proceedings.com/39213webtoc. pdf.

7. Vesga-Ferreira JC, Sierra JE, Vesga-Barrera JA. Modelling for TDMA under an AFR Scheme over Homeplug AV (HPAV). Indian Journal of Science and Technology. 2018; 11(3):1-9. https://doi.org/10.17485/ijst/2018/v11i3/117624.

8. Vesga Ferreira JC, Sierra JE, Vesga-Barrera JA. Performance evaluation under an OBA scheme CSMA / CA for HomePlug AV supported in Bianchis Model. Indian Journal of Science and Technology. 2018; 11(8):1-14. https://doi. org/10.17485/ijst/2018/v11i8/117511.

9. Latchman H, Katar S, Yonge L, Amarsingh A. High speed multimedia applications and Smart Energy PLC based on adaptations of HomePlug AV. IEEE 17th International Symposium on Power Line Communications and Its Applications; 2013. p. 143-8.

10. Kuo KC. The implementation of HomePlug AV system. International Conference on IC Design and Technology (ICICDT); 2016. p. 1-4.

11. Ferreira JC, Granados-Acuna G, Vesga-Barrera JA. Evaluation of the performance of a network LAN over powerline communications for the transmission of VoIP. Iteckne. 2016; 13(1):83-95. 
12. Vesga-Ferreira JC, Granados-Acuna G, Vesga-Barrera JA. Allocation of Medium Access Order Over Power Line Communications (PLC) Supported on Weighted Voting Games. Indian Journal of Science and Technology. 2017; 10(24):1-14. https://doi.org/10.17485/ijst/2017/ v10i24/102667.

13. Kemal MS, Olsen RL, Schwefel HP. Optimized Scheduling of Smart Meter Data Access for Real-Time Voltage Quality Monitoring. IEEE International Conference on Communications Workshops (ICC Workshops); 2018. p. $1-6$.
14. Valverde G, Zufferey T, Karagiannopoulos S, Hug G. Estimation of voltage sensitivities to power injections using smart meter data. IEEE International Energy Conference (ENERGYCON); 2018. p. 1-6. https://doi.org/10.1109/ ENERGYCON.2018.8398841.

15. Smart meters: what would it take to stop the national rollout juggernaut? [Internet]. [cited 2017 May 17]. Available from:https://eandt.theiet.org/content/articles/2017/05/ smart-meters-what-would-it-take-to-stop-the-nationalrollout-juggernaut/. 